\title{
Development of the micro pixel chamber based on MEMS technology
}

\author{
T. Takemura ${ }^{1}$,a, A. Takada ${ }^{1}$, T. Kishimoto ${ }^{1}$, S. Komura ${ }^{1}$, H. Kubo ${ }^{1}$, Y. Matsuoka ${ }^{1}$, K. Miuchi ${ }^{2}$, S. Miyamoto ${ }^{1}$, \\ T. Mizumoto ${ }^{1}$, Y. Mizumura ${ }^{1}$, T. Motomura ${ }^{3}$, Y. Nakamasu ${ }^{1}$, K. Nakamura ${ }^{1}$, M. Oda ${ }^{1}$, K. Ohta ${ }^{3}$, J. D. Parker ${ }^{4}$, \\ T. Sawano ${ }^{5}$, S. Sonoda ${ }^{1}$, T. Tanimori ${ }^{1}$, D. Tomono ${ }^{1}$, and K. Yoshikawa ${ }^{1}$ \\ ${ }^{1}$ Kyoto University, Department of Physics, Japan \\ ${ }^{2}$ Kobe University, Department of Physics, Japan \\ ${ }^{3}$ Dai Nippon Printing Co., Ltd. \\ ${ }^{4}$ CROSS-Tokai, Neutron R\&D Division, Japan \\ ${ }^{5}$ Kanazawa University, School of Mathematics and Physics, Japan
}

\begin{abstract}
Micro pixel chambers ( $\mu$-PIC) are gaseous two-dimensional imaging detectors originally manufactured using printed circuit board (PCB) technology. They are used in MeV gamma-ray astronomy, medicalimaging, neutron imaging, the search for dark matter, and dose monitoring. The position resolution of the present $\mu$-PIC is approximately $120 \mu \mathrm{m}$ (RMS), however some applications require a fine position resolution of less than $100 \mu \mathrm{m}$. To this end, we have started to develop a $\mu$-PIC based on micro electro mechanical system (MEMS) technology, which provides better manufacturing accuracy than PCB technology. Our simulation predicted the gains of MEMS $\mu$-PICs to be twice those of PCB $\mu$-PICs at the same anode voltage. We manufactured two MEMS $\mu$-PICs and tested them to study their behavior. In these experiments, we successfully operated the fabricated MEMS $\mu$-PICs and we achieved a maximum gain of approximately $7 \times 10^{3}$ and collected their energy spectra under irradiation of X-rays from ${ }^{55} \mathrm{Fe}$. However, the measured gains of the MEMS $\mu$-PICs were less than half of the values predicted in the simulations. We postulated that the gains of the MEMS $\mu$-PICs are diminished by the effect of the silicon used as a semiconducting substrate.
\end{abstract}

KEYWORDS: gaseous detector, Micro-pattern detector, Micro Electro Mechanical System

\section{Introduction}

A micro-pixel chamber ( $\mu$-PIC) [1] is a gaseous twodimensional imaging detector (Figure 1). Each pixel of the $\mu$-PIC, consisting of an anode via with a diameter of $60 \mu \mathrm{m}$ and a cathode electrode with a hole diameter of $250 \mu \mathrm{m}$, is positioned with a pitch of $400 \mu \mathrm{m}$. A gas avalanche occurs near the anode via because of the strong electric field between these electrodes; therefore, each pixel functions as a proportional counter. The anode vias are connected to an anode strip electrode on the back of the substrate, and the cathodes are formed in strip electrodes on the front surface. A two-dimensional readout is available using anode and cathode strips arranged orthogonally. $\mu$-PICs are manufactured by printed circuit board (PCB) technology and are composed of copper electrodes and 100- $\mu$ m-thick polyimide substrate. PCB technology enables the low-cost manufacturing of large-area $\mu$-PICs $\left(10 \times 10 \mathrm{~cm}^{2}\right.$ [2] or $\left.30 \times 30 \mathrm{~cm}^{2}[3]\right)$. PCB $\mu$-PICs feature a fine position resolution $(\mathrm{RMS} \approx 120 \mu \mathrm{m})$ [4], a high gas gain (nominal 6000, maximum 15000) [5], and good gain uniformity ( $\mathrm{RMS} \approx 5 \%$ ). $\mathrm{PCB} \mu$-PICs have been used in a wide range of applications including $\mathrm{MeV}$ gamma-ray astronomy [6], medical imaging [7], the search for dark matter [8], neutron imaging [9], and dose monitoring [10]. However, neu-

\footnotetext{
ae-mail: takemura@cr.scphys.kyoto-u.ac.jp
}

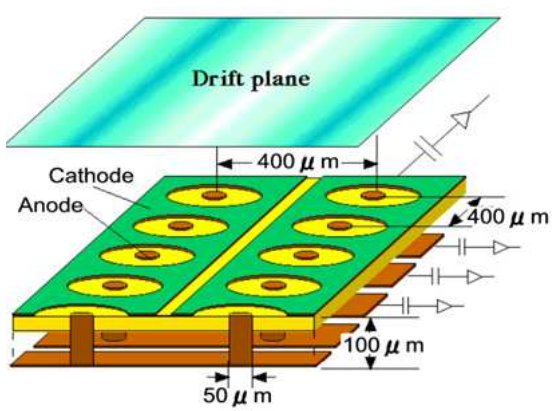

Figure 1. Schematic of a $\mu$-PIC based on PCB technology [1]. The $\mu$-PIC is composed of copper electrodes and a $100-\mu \mathrm{m}$ polyimide substrate. Each pixel of the $\mu$-PIC has an anode via with a diameter of $60 \mu \mathrm{m}$ and a cathode electrode with a hole diameter of $250 \mu \mathrm{m}$ and is fabricated with a pitch of $400 \mu \mathrm{m}$. A gas avalanche occurs near the anode via because of the strong electric field between these electrodes; therefore, each pixel functions as a proportional counter.

tron imaging applications require a position resolution of less than $100 \mu \mathrm{m}$ and $\mathrm{MeV}$ gamma-ray astronomy requires precise tracking of charged particles with an angular resolution of less than $5^{\circ}$. Satisfying these requirements ne- 
Table 1. Thickness and relative permittivity values used in simulations of each layer in the $\mu$-PICs.

\begin{tabular}{llll}
\hline material & $\begin{array}{l}\text { relative } \\
\text { permittivity }\end{array}$ & $\begin{array}{l}\text { MEMS } \\
{[\mu \mathrm{m}]}\end{array}$ & $\begin{array}{l}\text { PCB } \\
{[\mu \mathrm{m}]}\end{array}$ \\
\hline copper electrode & $10^{10}$ & 15 & 15 \\
polyimide & 3.2 & 4 & $\sim 100$ \\
silicon & 11 & 400 & - \\
$\mathrm{SiO}_{2}$ & 4.5 & 1 or 10 & - \\
\hline
\end{tabular}

cessitates the development of a $\mu$-PIC with a fine position resolution.

Because of the limitation of the manufacturing accuracy of PCB technology, the diameter of $\mu$-PIC anode vias is limited to approximately $50 \mu \mathrm{m}$. Therefore, a more advanced technology is needed for manufacturing fine-pitch $\mu$-PICs. Micro electro mechanical system (MEMS) technology is one such capable technology. MEMS technology is well established, and MEMS manufacturing costs have been reduced to level similar to those of PCB technology. In addition, MEMS technology enables the use of thicker substrates compared with those for PCB technology. In the case of $\mu$-PICs, the gain depends on the thickness of the substrate: a two fold thicker substrate improves the gain by a factor of two [11]. Thus, a MEMS $\mu$-PIC is expected to exhibit a fine position resolution and a high gas gain. However, the substrates used in MEMS technology comprise silicon, which is a semiconductor; thus, the effect of a semiconducting substrate being substituted for the insulating substrate of $\mu$-PICs should be investigated. In this work, we manufactured two MEMS $\mu$-PICs to investigate their behavior. Here, we report the results of both the simulations and experiments.

\section{Simulation study of MEMS $\mu$-PIC}

We fabricated a MEMS $\mu$-PIC with a structure similar to that of a PCB $\mu$-PIC to focus on the effect using a semiconducting instead of an insulating substrate. Figure 2 shows a photo and a schematic of the cross-section of the manufactured MEMS $\mu$-PIC. The differences between a PCB $\mu$-PIC and a MEMS $\mu$-PIC lie in the substrate material and its thickness, which are listed in Table 1. For simulation purpose, the MEMS $\mu$-PIC substrate was assumed to comprise a $400-\mu$ m-thick silicon layer, a thin $\mathrm{SiO}_{2}$ layer, and a thin polyimide layer; by contrast, the PCB $\mu$-PIC substrate was assumed to be composed of a polyimide layer with a thickness of $100 \mu \mathrm{m}$. The thin $\mathrm{SiO}_{2}$ layer and polyimide layer are insulated on MEMS $\mu$-PICs to enhance their discharge tolerance. We varied the thickness of the $\mathrm{SiO}_{2}$ layer in the manufactured MEMS $\mu$-PICs to study its effect on device performance. Other parameters (such as the thickness of the strips, pitches of pixels, diameter of the cathode hole, diameter of the anode via, and width of a cathode strip) were approximately the same.

We evaluated the geometries of the PCB and MEMS $\mu$-PICs to study their gains. We fabricated $\mu$-PIC pixel electrodes using the Gmsh program [12] and calculated the electric field around the $\mu$-PICs using the Elmer pro-
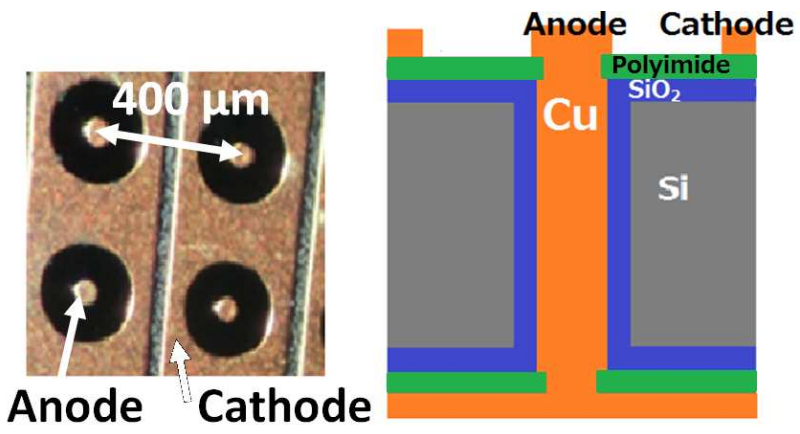

Figure 2. Photograph (left) and schematic of the cross-section (right) of a MEMS $\mu$-PIC. The substrate of the MEMS $\mu$-PIC comprises to a silicon layer with a thickness of $400 \mu \mathrm{m}$, a thin $\mathrm{SiO}_{2}$ layer, and a thin polyimide layer. The thin $\mathrm{SiO}_{2}$ and polyimide layers are insulated on the MEMS $\mu$-PIC to enhance the discharge tolerance.
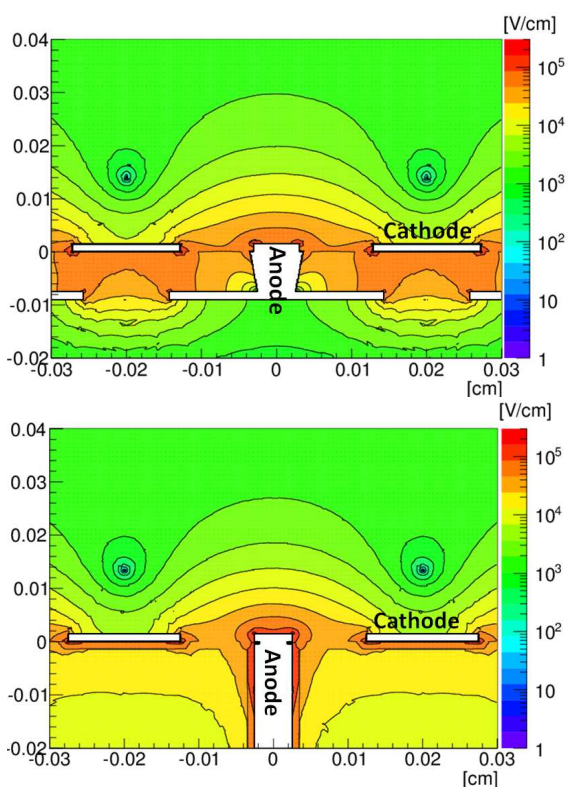

Figure 3. Electric fields of the cross section of a PCB $\mu$-PIC and a MEMS $\mu$-PIC calculated using the program Elmer for the case of an anode voltage of $460 \mathrm{~V}$. The drift electric field and cathode voltage were fixed at $1.0 \mathrm{kV} / \mathrm{cm}$ and $0 \mathrm{~V}$, respectively.

gram [13]. We simulated the avalanche and obtained gas gains using the Garfield++ program with a threedimensional mesh [14]. We used a gas of $\mathrm{Ar} / \mathrm{C}_{2} \mathrm{H}_{6}$ (pressure ratio 90:10) at $1 \mathrm{~atm}$ and adopted a penning-effect rate of 0.31 [15]. The drift electric field and cathode voltage were fixed at $1.0 \mathrm{kV} / \mathrm{cm}$ and $0 \mathrm{~V}$, respectively. Figure 3 shows the electric fields of the cross sections of the PCB $\mu$-PIC and MEMS $\mu$-PIC for the case of an anode voltage of $460 \mathrm{~V}$. The electric field at $1 \mu \mathrm{m}$ above the edge of the anode via was $1.7 \times 10^{5} \mathrm{~V} \mathrm{~cm}^{-1}$ for the PCB $\mu$-PIC and $2.2 \times 10^{5} \mathrm{~V} \mathrm{~cm}^{-1}$ for the MEMS $\mu$-PIC. The thick substrate of the MEMS $\mu$-PIC concentrates the electric field lines to an anode via, because the thick substrate obstructs the electric field lines between the cathode and anode strips. Therefore, the MEMS $\mu$-PIC exhibits a stronger electric 


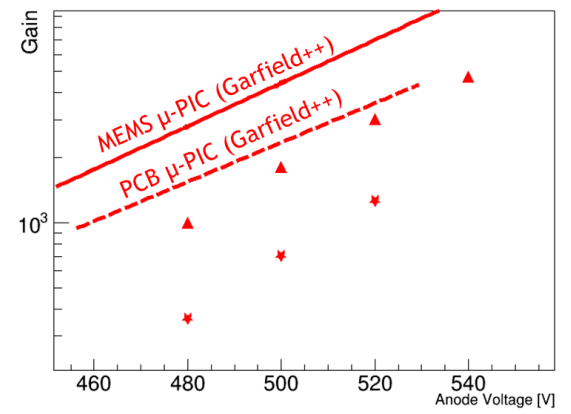

Figure 4. The simulated and measured gains of $\mathrm{PCB}$ and MEMS $\mu$-PICs. The dashed and solid lines represent the simulated gains of a PCB $\mu$-PIC and a MEMS $\mu$-PIC, respectively. The simulation predicted the gains of the MEMS $\mu$-PIC are to be twice those of the PCB $\mu$-PIC at the same anode voltage. Stars and triangles represent the measured gains of the MEMS $\mu$-PIC with $1-\mu \mathrm{m}$ and $10-\mu \mathrm{m} \mathrm{SiO}_{2}$ layers, respectively. The gains were measured using the setup shown in Figure 5.

field. The dependence of the simulated avalanche size on the anode voltage is shown in Figure 4. In this figure, the dashed and solid lines represent the simulated gains of the PCB [16] and MEMS $\mu$-PICs, respectively. The measured gains of the PCB $\mu$-PICs agree well the simulated gains [16]; we therefore considered Garfield ++ to be a reliable simulator of an avalanche around a $\mu$-PIC anode. The simulation predicted the gains of the MEMS $\mu$-PIC to be twice those of the PCB $\mu$-PIC at the same anode voltage. In addition, we studied the effect of the $\mathrm{SiO}_{2}$ layer in the simulation, and observed that the gains of the MEMS $\mu$-PIC were independent of the thickness of the $\mathrm{SiO}_{2}$ layer when the layer thickness was less than $15 \mu \mathrm{m}$.

\section{Measurements of the gas gains of MEMS $\mu$-PICs}

To confirm the simulation results, we operated the manufactured MEMS $\mu$-PICs using the setup shown in Figure 5. The active area of the MEMS $\mu$-PIC was $5 \times$ $10 \mathrm{~mm}^{2}$, and it comprised 6 cathode and 10 anode strips. The MEMS $\mu$-PIC was placed in a sealed aluminum vessel filled with $\mathrm{Ar} / \mathrm{C}_{2} \mathrm{H}_{6}$ gas (in a pressure ratio of 90:10) at atmospheric pressure. We positioned a gas electron multiplier (GEM) [17] $3 \mathrm{~mm}$ above the $\mu$-PIC. The GEM has a polyimide substrate with a thickness of $100 \mu \mathrm{m}$, and its hole diameter is $70 \mu \mathrm{m}$ with a pitch of $140 \mu \mathrm{m}$; and the active area of the GEM is $10 \times 10 \mathrm{~cm}^{2}$. We operated the GEM at a gain of approximately 20; the electric field of the induction field was $1 \mathrm{kV} / \mathrm{cm}$. The drift plane was 3 $\mathrm{mm}$ above the GEM, and the electric field of $250 \mathrm{~V} / \mathrm{cm}$ was applied in the drift space. Therefore, the volume of the detection space was $5 \times 10 \times 3 \mathrm{~mm}^{3}$; this space is indicated by the hatched area in Figure 5. The signals from anode and cathode strips were read out by a CMOS ASIC chip [18]. This ASIC has 16 analog input channels, 16 digital output channels and an analog-sum signal. The analog-sum signal is fed to an 8-bit FADC operated at 25

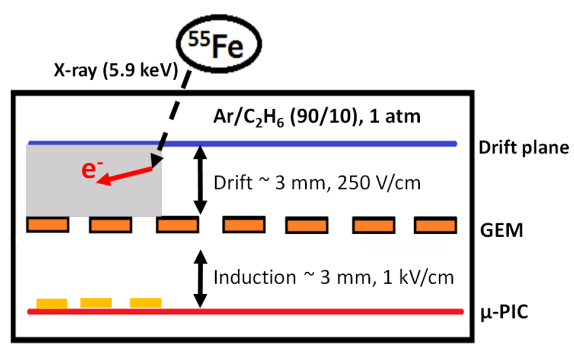

Figure 5. Schematic of the setup of the test operation. The details are described in the text.

MHz. The details of the data acquisition system used in these experiments have been described elsewhere [18].

We first operated the MEMS $\mu$-PIC with a $10-\mu \mathrm{m} \mathrm{SiO}_{2}$ layer under irradiation of $5.9 \mathrm{keV}$ X-rays from ${ }^{55} \mathrm{Fe}$. Figure 6 shows the signal when anode voltage was $460 \mathrm{~V}$. The MEMS $\mu$-PIC was stably operated at a discharge rate of less than $0.03 \mathrm{~Hz}$ and at the anode voltage of $560 \mathrm{~V}$. In the case of the MEMS $\mu$-PIC with $1-\mu$ m-thick $\mathrm{SiO}_{2}$ layer, the leak current was approximately $160 \mathrm{nA}$ under the applied anode voltage; the leak current remained greater than $20 \mathrm{nA}$ for longer than $4 \mathrm{~h}$. The discharge rate of the MEMS $\mu$-PIC with a $1-\mu$ m-thick $\mathrm{SiO}_{2}$ layer was greater than $0.5 \mathrm{~Hz}$ at an anode voltage of $530 \mathrm{~V}$. Therefore, $\mathrm{SiO}_{2}$ layer is essential for its stable operation.

Figure 7-(a) and (b) show the ${ }^{55} \mathrm{Fe}$ energy spectra of PCB $\left(25 \times 25 \mathrm{~mm}^{2}\right)$ and MEMS $\mu$-PICs, respectively. The energy resolution of the PCB $\mu$-PIC at $5.9 \mathrm{keV}$ was $40 \%$, with a gain of $1.1 \times 10^{3}$; by contrast the energy resolution of the MEMS $\mu$-PIC (1- $\mu$ m-thick $\mathrm{SiO}_{2}$ layer) was $5.9 \mathrm{keV}$ was $57 \%$ with a gain of $1.2 \times 10^{3}$. The energy resolution of the MEMS $\mu$-PIC (10- $\mu$ m-thick $\mathrm{SiO}_{2}$ layer) was nearly equal to that of the MEMS $\mu$-PIC (1- $\mu$ m-thick $\mathrm{SiO}_{2}$ layer). We considered that the poor energy resolution observed for the MEMS $\mu$-PICs as compared to the PCB $\mu$-PIC is due to the detection area, which was $10 \%$ smaller than that of the PCB $\mu$-PIC. The electrons escaped from the smaller detection area more easily, depositing only partial energy and, thus, leading to worsened energy resolution. In future experiments, we plan to obtain the energy spectrum with imaging for the rejection of escape electrons, after we achieve stable operation with a gain of $10^{4}$.

In Figure 4, stars and triangles represent the measured gains of MEMS $\mu$-PIC with $1-\mu \mathrm{m}$ - and $10-\mu \mathrm{m}$-thick $\mathrm{SiO}_{2}$ layers, respectively. The maximum gain of the MEMS $\mu$-PIC reached approximately $7 \times 10^{3}$. The gains of the MEMS $\mu$-PICs of $1-\mu$ m- and $10-\mu$ m-thick $\mathrm{SiO}_{2}$ layers were $16 \%$ and $40 \%$ that of the simulated gains at an anode voltage of $500 \mathrm{~V}$, respectively. Because semiconductors contain charge carriers, the silicon near the anodes and cathodes became negatively and positively charged, respectively under an applied anode voltage. However, our simulations with the Elmer and Garfield++ programs did not account for such an effect. The electric field around the anode via would be weaker than that predicted by the simulations because of the charge-up effect of silicon; there- 


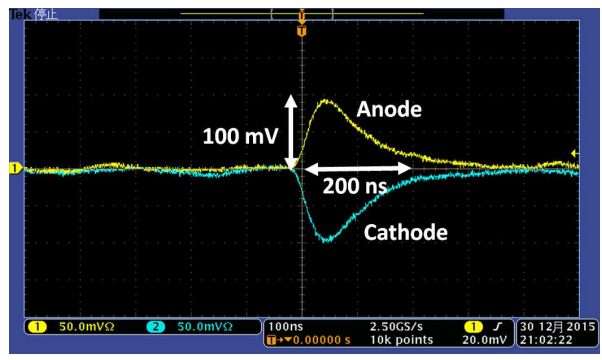

Figure 6. The signals of MEMS $\mu$-PIC with $\mathrm{SiO}_{2}$ layer of $10 \mu \mathrm{m}$ by the $5.9 \mathrm{keV} \mathrm{X}$-ray of ${ }^{55} \mathrm{Fe}$ source. Positive and negative signals are obtained from cathodes and anodes, respectively. Voltage between GEM top and bottom is $320 \mathrm{~V}$, and anode voltage is $460 \mathrm{~V}$. The filled gas is with a normal-pressure $\mathrm{Ar} / \mathrm{C}_{2} \mathrm{H}_{6}$ (pressure ratio 90:10) gas mixture.
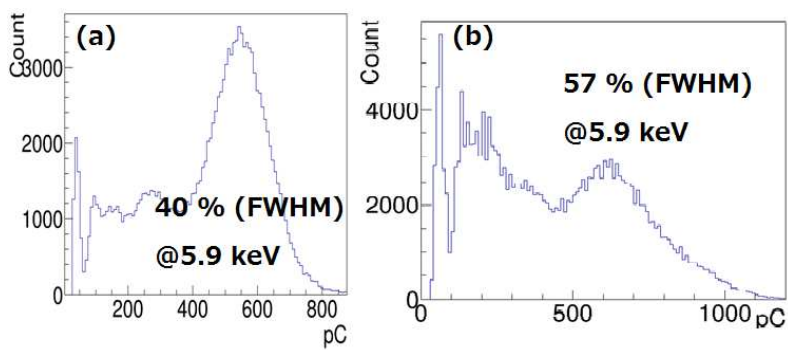

Figure 7. The energy spectrum of the $5.9 \mathrm{keV}$ X-ray of ${ }^{55} \mathrm{Fe}$ source (a)PCB $\mu$-PIC and (b)MEMS $\mu$-PIC, respectively.

fore, the gains of the MEMS $\mu$-PIC were reduced. In addition, the measured gas gain depends on the thickness of the $\mathrm{SiO}_{2}$ layer, which served as an insulator positioned between the electrodes and the silicon substrate. As described in section 2 , the simulated gain is independent of the thickness of the $\mathrm{SiO}_{2}$ layer; however, thinner $\mathrm{SiO}_{2}$ layer results in a greater charge-up effect. The leak current during startup is additional evidence of this charge-up effect.

To achieve both stable operation and a high gain with a MEMS $\mu$-PIC, we need to reduce the charge-up effect of the silicon. We considered two solutions to this issue. One is to fabricate a MEMS $\mu$-PIC using a glass substrate that contains no semiconductor. We can manufacture a MEMS $\mu$-PIC on a glass substrate, but the cost will be expensive. The other solution is to fabricate a MEMS $\mu$-PIC with a thick $\mathrm{SiO}_{2}$ layer. We believe that we can fabricate a MEMS $\mu$-PIC with a $\mathrm{SiO}_{2}$ layer with $15-30-\mu$ m-thick, and we plan to manufacture them in the future. We expect that the resulting MEMS $\mu$-PICs will be useful in various applications.

\section{Summary}

The applications of $\mu$-PICs require a finer position resolution than that of current PCB $\mu$-PICs. To satisfy this requirement, we fabricated and studied MEMS $\mu$-PICs. Specifically, we fabricated MEMS $\mu$-PICs with a structure similar to that of a PCB $\mu$-PIC to focus on the effects of using semiconducting instead of an insulating substrate.
Simulations predicted the gains of the MEMS $\mu$-PICs to be twice those of PCB $\mu$-PICs at the same anode voltage. The MEMS $\mu$-PIC with a $1-\mu$ m-thick $\mathrm{SiO}_{2}$ layer was unstable, demonstrating that a $\mathrm{SiO}_{2}$ layer is essential to the stable operation of MEMS $\mu$-PICs. For the first time, we successfully obtained a signal and energy spectra using MEMS $\mu$-PICs under irradiation of $\mathrm{X}$ rays from ${ }^{55} \mathrm{Fe}$. The maximum gain of the MEMS $\mu$-PICs reached approximately $7 \times 10^{3}$. However, the gains measured at an anode voltage of $500 \mathrm{~V}$ were $16-40 \%$ of that of the simulated values. We considered that the gains of the MEMS $\mu$-PICs were diminished by the effect of silicon as a semiconductor. To minimize the effect of the semiconductor, we plan to manufacture MEMS $\mu$-PICs with thicker $\mathrm{SiO}_{2}$ layers or glass substrates.

\section{Acknowledgements}

This work was supported by the "UCHUGAKU" project of the Unit of Synergetic Studies for Space, Kyoto University.

\section{References}

[1] A. Ochi, et al., Nuclear Instruments and Methods in Physics Research Section A 471, 264-276 (2001).

[2] H. Kubo, et al., Nuclear Instruments and Methods in Physics Research Section A 513, 94-98 (2003).

[3] A. Takada, et al., Nuclear Instruments and Methods in Physics Research Section A 573, 195-199 (2007).

[4] T. Nagayoshi, et al., Nuclear Instruments and Methods in Physics Research Section A 525, 20-27 (2004).

[5] K. Miuchi, et al., IEEE Transactions on Nuclear Science 50, 825-830 (2003).

[6] T. Tanimori, et al., The Astrophysical Journal 810, 28 (2015).

[7] S. Kabuki, et al., Nuclear Instruments and Methods in Physics Research Section A 623, 606-607 (2010).

[8] K. Nakamura, et al., Progress of Theosetical and Experimental Physics, 043F01 (2015).

[9] J. D. Parker, et al., Nuclear Instruments and Methods in Physics Research Section A 697, 23-31 (2013).

[10] T. Mizumoto, et al., Journal of Instrumentation 10, C06003 (2015).

[11] T. Nagayoshi, et al., Nuclear Instruments and Methods in Physics Research Section A 546, 457-465 (2005).

[12] http://geuz.org/gmsh.

[13] http://www.csc.fi/english/pages/elmer.

[14] http://garfieldpp.web.cern.ch/garfieldpp.

[15] Ö. Şahin, et al., Journal of Instrumentation 5, P05002 (2010).

[16] A. Takada, et al., Journal of Instrumentation 8 , C10023 (2013).

[17] F. Sauli, Nuclear Instruments and Methods in Physics Research Section A 386, 531-534 (1997).

[18] T. Mizumoto, et al., Nuclear Instruments and Methods in Physics Research Section A 800, 40-50 (2015). 